Introduction The presence of antibodies against Chlamydia trachomatis $(\mathrm{Ct})$ is indicative of previous genital or ocular infection. Serology was introduced in the 1970s to support the diagnosis of pelvic inflammatory disease (PID), but fell out of favour due to its cross-reactivity with Chlamydophila (Chlamydia) pneumoniae bacteria. With the recent development of sensitive and specific assays, as well as the identification of immunogenic Ct antigens developed as recombinant proteins, serology holds the potential to be a useful tool in public health. To date, there has been no summary of the techniques used, their development and their potential usefulness in public and tropical medicine.

Methods We searched PubMed, Cochrane, Lilacs, Scielo, Scopus and Web of Science for articles published on serological techniques and their use in a public health context. Studies were categorised by technique employed, antigen used and antibody detected.

Results A total of 16 studies were included- 5 related to ocular Ct infection and 11 related to genital $\mathrm{Ct}$ infection. The trachoma studies were predominantly based on Tanzanian samples, while the genital studies were based on samples from an array of countries. The studies were heterogeneous in design, assay and antigen used, and immunoglobulin detected. The estimated prevalence of antibodies against $\mathrm{Ct}$ in trachoma studies ranged from $0 \%-62 \%$; from $0 \%-88.9 \%$ in genital studies. For genital Ct infections, serology is commonly used to explore disease sequelae. For ocular Ct infections, serology is explored as a means to monitor elimination efforts.

Conclusion Techniques used to measure the prevalence of antibodies against $\mathrm{Ct}$ have reported increased sensitivity and specificity. There is wide diversity in antigens and assays used and antibodies detected. The practicality of an assay depends on resources available, purpose of the study, and population being studied. There is wide scope for the development and refinement of techniques to increase the value of serology as it relates to development of new techniques, research and public health.

\section{P3.215 CHARACTERISE THE TEMPORAL EVOLUTION OF HIV INCIDENCE AMONG STABLE COUPLES IN SUB-SAHARAN AFRICA}

Susanne Awad, Hiam Chemaitelly, Laith J Abu-Raddad. Weill Cornell Medicine-Qatar, Doha, Qatar

10.1136/sextrans-2017-053264.450

Introduction We quantified and characterised the temporal evolution of the contribution of several types of sexual-partnership HIV statuses to total HIV incidence in representative countries at different HIV epidemic scales in sub-Saharan Africa.

Methods A pair-based mathematical model was constructed to accommodate for movement of individuals between different forms of sexual partnerships and HIV infection statuses. Stable couples (SCs) were divided into sero-concordant negative (SCNCs), sero-discordant (SDCs), and sero-concordant positive couples. Throughout epidemic phases, historical and future trends (1980-2030) of sources of HIV incidence by partnership status were projected based on nationally-representative epidemiologic and demographic data.

Results As the epidemics emerged and peaked, HIV incidence resulting among SDCs increased from $6 \%-32 \%$ to $19 \%-44 \%$ of total HIV incidence, mostly due to transmissions within the SDCs. As the epidemics started their natural and further decline with antiretroviral therapy (ART) scale-up, this proportion declined in intermediate and high HIV-prevalence countries, but increased in low HIV-prevalence countries. As the epidemics emerged and peaked, HIV incidence resulting among SCNCs from extramarital sex declined from 54\%-80\% to $35 \%-73 \%$ of total HIV incidence. As the epidemics started their decline, this proportion increased in intermediate and high HIV-prevalence countries, but declined in low HIV-prevalence countries. HIV incidence resulting among individuals not in SCs was 9\%-29\% of total HIV incidence and was stable throughout the epidemics, but larger with higher HIV prevalence.

Conclusion The contribution of different forms of sexual-partnership HIV statuses to total HIV incidence was dependent on HIV epidemic phase and scale. Throughout the epidemics, more than two thirds of HIV incidence occurred among individuals engaged in SCs. The majority of incidence among SCs was due to extramarital sex. To achieve reductions in HIV incidence, prevention approaches should target both SCs and individuals not in SCs.

\section{P3.216 ASSESSING HPV GENOTYPE PREVALENCE IN INFECTION AND DISEASE IN YOUNG AUSTRALIAN WOMEN FOLLOWING THE INTRODUCTION OF A NATIONAL VACCINATION PROGRAM}

${ }^{1}$ Suzanne Garland, ${ }^{2} \mathrm{AM}$ Cornall, ${ }^{3} \mathrm{JML}$ Brotherton, ${ }^{4} \mathrm{SL}$ Osborne, ${ }^{5} \mathrm{CD}$ Wrede, ${ }^{6} \mathrm{JD}$ Wark ${ }^{3} \mathrm{M}$ Saville, ${ }^{7} \mathrm{~J}$ Pyman, ${ }^{8} \mathrm{~J}$ Tan, ${ }^{9} \mathrm{ET}$ Callegari, ${ }^{3} \mathrm{DM}$ Gertig, ${ }^{10} \mathrm{Y}$ Jayasinghe, ${ }^{11} \mathrm{M}$ Malloy, ${ }^{12} \mathrm{M}$ Pitts, ${ }^{1} \mathrm{SN}$ Tabrizi. ${ }^{1}$ Royal Women's Hospital, Department of Microbiology and Infectious Diseases, Parkville, Australia; ${ }^{2}$ Royal Women's Hospital, Department of Microbiology and Infectious Diseases, and Murdoch, Parkville, Australia; ${ }^{3}$ National HPV Vaccination Program Register, VCS, East Melbourne, Australia; ${ }^{4}$ Royal Women's Hospital, Department of Microbiology and Infectious Diseases, Parkville, and Murdoch, Parkville, Australia; ${ }^{5}$ Royal Women's Hospital, Parkville, Australia; ${ }^{6}$ The University of Melbourne, Parkville, Australia; ${ }^{7}$ Department of Anatomical Pathology, The Royal Women's Hospital, Parkville, Australia; ${ }^{8}$ Department of Obstetrics and Gynaecology, University of Melbourne and Oncology and Dysplasia Unit, R, Parkville, Australia; ${ }^{9}$ The University of Melbourne, Department of Medicine, Royal Melbourne Hospital, Parkville, Australia; ${ }^{10}$ Department of Obstetrics and Gynaecology, University of Melbourne, Department of Gynaecology, Roya, Parkville, Australia; ${ }^{11}$ National Hpv Vaccination Program Register, VCS, Parkville, Australia: ${ }^{12}$ Australian Research Centre in Sexual Health and Society, La Trobe University, Melbourne, Australia

10.1136/sextrans-2017-053264.451

Introduction The National HPV Vaccination Program, utilising the quadrivalent HPV vaccine, was implemented in Australia in 2007. As the first country to introduce this scheme, Australia is ideally placed to identify changes in HPV genoprevalence in a vaccinated population, as well as in cervical intraepithelial neoplasia grade 3 (CIN3) lesions.

Methods The VACCINE (Vaccine Against Cervical Cancer Impact and Effectiveness) study was designed to assess prevalence of vaccine-targeted HPV genotype infections. In substudy A, sexually active Victorian women aged 18-25 years, recruited through targeted social media advertising on Facebook, were asked to complete an online questionnaire and provide a self-collected vaginal swab for HPV DNA genotyping. The National HPV Vaccination Program Register (NHVPR) was utilised to verify self-reported vaccination status. In sub-study B, causal HPV genotypes in 529 CIN3 cases among vaccine-eligible young women were determined using 
laser capture microdissection and compared with pre-vaccine era data.

Results In sub-study A, 1095 participants were recruited (18\% were virginal so no swab was collected) and 774 (77\%) completed all study requirements. Vaccine-targeted HPV genotypes were detected in only 13 samples (1.7\%); 11 HPV16 and two HPV6: none were in participants vaccinated prior to sexual debut. In sub-study B, 333 women (62.9\%) had a HPV16/18 positive CIN3 detected. The pre-vaccine era population included 98 women aged 18-25, and 115 women aged 2632 , recruited between 2001 and 2005. Compared to the prevaccine cohort, the proportion of HPV16/18 positive CIN3 was significantly lower in the post-vaccine 18-25 year old group $(71.4 \%$ vs $54.9 \%, \mathrm{p}=0.009)$, but was not significantly different in the $26-32$ year old age group $(61.7 \%$ vs $65.9 \%$, $\mathrm{p}=0.409$ ).

Conclusion The VACCINE study demonstrates that vaccinerelated HPV genotype prevalence is remarkably low amongst vaccine-eligible Victorian women, and also demonstrates a reduction in the proportion of CIN3 caused by vaccine preventable HPV types in younger, vaccine-eligible women.

Support: I Professor Suzanne Garland, have received Grants to my institution from Commonwealth Department of Health for HPV genoprevalance surveillance post vaccination, Merck and GSK (GlaxoSmithKline) to perform phase 3 clinical vaccine trials: Merck to evaluate HPV in RRP post vaccination programme, CSL for HPV in cervical cancer study, and VCA (Victoria Cancer Agency) for a study on effectiveness of public bealth HPV vaccine study plus a study on associations of early onset cancers. I have received speaking fees from MSD and SPMSD for work performed in my personal time. Merck paid for travel and accommodation to present at HPV Advisory board meetings.

\section{P3.217 HUMAN IMMUNODEFICIENCY VIRUS (HIV) SERODISCORDANCE AND ASSOCIATED FACTORS AMONG HIV POSITIVE CLIENTS ACCESSING ANTI- RETROVIRAL THERAPY IN NIGERIA}

${ }^{1}$ Temitope 'Wunmi Ladi-Akinyemi, ${ }^{2}$ Babatunde Oladipupo Ladi-Akinyemi, ${ }^{3}$ Olusegun Ige Adebisi, ${ }^{1}$ Adekemi Oluyemisi Sekoni. ${ }^{1}$ University of Lagos, College of Medicine, Lagos, Nigeria; ${ }^{2}$ APIN Public Health Initiative, Ibadan, Nigeria; ${ }^{3}$ Olabisi Onabanjo University Teaching Hospital, Sagamu, Nigeria

\subsection{6/sextrans-2017-053264.452}

Introduction In Africa, there is increasing evidence that a large proportion of new HIV infections occur in cohabitating couples, many of whom are unaware of both partners' serostatus. Sub-Saharan Africa has the highest prevalence and incidence of HIV infection worldwide, mostly attributable to heterosexual transmission. In Nigeria, the bulk of the new infections occur in persons who are not engaging in high risk sex, a sub-population that includes cohabiting or married sexual partners. This study was undertaken to determine the prevalence and assess factors associated with HIV serodiscordance status among HIV positive patients accessing ART in secondary health facilities in Ogun State, South West Nigeria.

Methods This is a descriptive cross-sectional study conducted in four secondary health facilities offering HIV services in Ogun State over a six months period. Sample size of 650 was determined using the Cochrane formula and the respondents were consecutively recruited into the study. Data was collected using a structured interviewer administered questionnaire and analysis was done using the statistical package for social sciences (SPSS) version 17.0. Results was presented using frequency tables, tests of associations was done using the Chi-square test and multiple Logistic regression with level of significance set at 0.05 .

Results A total of 637 questionnaires was retrieved (response rate 98\%). Two hundred and Seventy eight (43.6\%) of the respondents did not know their spouse HIV status while 359 knew partners status. Of the 359, 166 clients are serodiscordant (46.2\%) while $193(53.8 \%)$ were seroconcordant. $50.7 \%$ were aged 30-39 years, the mean age was $35.8 \pm 9.1$ years. Significantly more females $(74.7 \%)$ than males $(25.3 \%)$ had serodiscordant partners ( $p$-value $=0.002$ ). Clients who did not consume alcohol were significantly more likely to have serodiscordant partners compared with clients who consumed alcohol $(p$-value $=0.001)$. Serodiscordant partners were significantly more among clients with infrequent sexual activities compared with clients with frequent sexual activities ( $\mathrm{p}$-value $=0.001$ ). Predictors of serodiscordance status among partners were clients who were $<35$ years old (odd ratio $(\mathrm{OR})=1.727$, confidence interval $(\mathrm{CI})=1.107-2.693)$ and did not consumed alcohol $(\mathrm{OR}=2.204, \mathrm{CI}=1.070-4.542)$, with partners whose occupation were skilled $(\mathrm{OR}=7.159, \mathrm{CI}=1.440-35.604)$ and semi-skilled $(\mathrm{OR}=6.189, \mathrm{CI}=1.216-31.503)$ and clients with infrequent sexual activities $(\mathrm{OR}=3.182, \mathrm{CI}=1.437-7.046)$.

Conclusion The study indicates that HIV positive clients could have serodiscordant partners if they could modify their lifestyle, have infrequent sexual activities (Not more than twice in a month) with partners that are gainfully engaged. It is therefore recommended that for early identification of at risk partners, couple HIV counselling and testing needs to be scaled up in addition to group-based interventions in order to pick serodiscordant partners early and provided all the necessary behavioural change support required to remain serodiscordant.

\section{P3.218 DEMOGRAPHIC AND CLINICAL PROFILES OF WOMEN WITH BACTERIAL VAGINOSIS AND SEXUALLY TRANSMITTED INFECTIONS: IMPLICATIONS FOR THE MANAGEMENT OF VAGINAL DISCHARGE SYNDROME IN SOUTH AFRICA} ${ }^{1}$ Ranmini Kularatne. 'National Institute of Communicable Diseases, Johannesburg, South African Republic; ${ }^{2}$ Institute of Communicable Diseases, Johannesburg, South African Republic

\subsection{6/sextrans-2017-053264.453}

Introduction Current South African syndromic management guidelines for vaginal discharge syndrome (VDS), recommend only metronidazole and clotrimazole therapy for women 35 years or older, whose partners have no urethral discharge. This guidance assumes the older women have bacterial vaginosis or candidiasis without STIs and that their partners are not asymptomatic. We describe demographic, behavioural and clinical characteristics of women with VDS comparing those with BV and candida to those infected with STI pathogens.

Methods Cross sectional study, in which women $\geq 18$ years presenting with VDS to five primary healthcare centres between January 2015 - September 2016 were enrolled. Demographic and clinical data were collected using a nurse-administered questionnaire. Vaginal smears, endocervical swabd and venous blood specimens were collected for laboratory testing. Presence 